\title{
Optical evaluation of digital micromirror devices (DMDs) with UV-grade fused silica, sapphire, and magnesium fluoride windows and longterm reflectance of bare devices
}

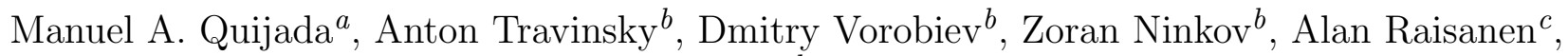 \\ Massimo Robberto ${ }^{d}$, and Sara Heap ${ }^{a}$ \\ ${ }^{a}$ NASA Goddard Space Flight Center, Optics Branch, \\ 8800 Greenbelt Rd., Greenbelt, MD 20771; \\ ${ }^{b}$ Center for Imaging Science, 54 Lomb Memorial Drive, Rochester, NY; \\ ${ }^{c}$ Department of Manufacturing and Mechanical Engineering Technology, \\ Rochester Institute of Technology, Rochester NY 14623; \\ ${ }^{d}$ Space Telescope Sciences Institute, \\ 3700 San Martin Dr., Baltimore, MD 21218;
}

\begin{abstract}
Digital micromirror devices (DMDs) are commercial micro-electromechanical systems, consisting of millions of mirrors which can be individually addressed and tilted into one of two states $\left( \pm 12^{\circ}\right)$. These devices were developed to create binary patterns in video projectors, in the visible range. Commercially available DMDs are hermetically sealed and extremely reliable. Recently, DMDs have been identified as an alternative to microshutter arrays for space-based multi-object spectrometers (MOS). Specifically, the MOS at the heart of the proposed Galactic Evolution Spectroscopic Explorer (GESE) uses the DMD as a reprogrammable slit mask. Unfortunately, the protective borosilicate windows limit the use of DMDs in the UV and IR regimes, where the glass has insufficient throughput. In this work, we present our efforts to replace standard DMD windows with custom windows made from UV-grade fused silica, low-absorption optical sapphire (LAOS) and magnesium fluoride $\left(\mathrm{MgF}_{2}\right)$. We present transmission measurements of the antireflection coated windows and the reflectance of bare (window removed) DMDs. Furthermore, we investigated the long-term stability of the DMD reflectance and experiments for coating DMD active area with a layer of pure aluminum (Al) to boost reflectance performance in the UV spectral range $(200-400 \mathrm{~nm})$.
\end{abstract}

Keywords: Digital Micromirror Device, reflectance, transmittance, Galactic Evolution Spectroscopic Explorer, digital mcromirror array, scattering, DMD, MOS

\section{INTRODUCTION}

The study of the possible drivers for galaxy evolutions (e.g. accretion, mergers, star formation, stellar evolution and feedback, growth of black holes, etc) are some of the most outstanding questions in cosmology. A large spectroscopic survey, which will be essential to answer the question of galaxy evolution, will require a multiobject spectrograph (MOS) capable of recording the spectra of hundreds of galaxies in a single exposure. The MOS must have adjustable slits to eliminate confusion with nearby sources and to block out unwanted zodiacal background, which would otherwise swamp the light from these faint galaxies. The MOS should have access to the far-ultraviolet (FUV) $(120-200 \mathrm{~nm})$ radiation emitted by a $\mathrm{z} \approx 1$ galaxy because this spectral region has a rich set of diagnostics of stars, gas, and dust in the galaxy. Access to the blue-red spectral regions (400-700 nm) is also essential for determining the precise redshift of a galaxy, its stellar mass, and abundances of the elements, and for characterizing dust extinction. Because the light from $\mathrm{a} z \approx 1$ galaxy is redshifted before reaching us, a large spectroscopic survey should be sensitive over the spectral interval of $200-1600 \mathrm{~nm}^{1}$ One of the main constraints that has prevented progress in performing large surveys of galaxies is because there is no multi-object

Further author information: (Send correspondence to M.A.Q.: E-mail: manuel.a.quijada@nasa.gov, Telephone: +1 3012863544 


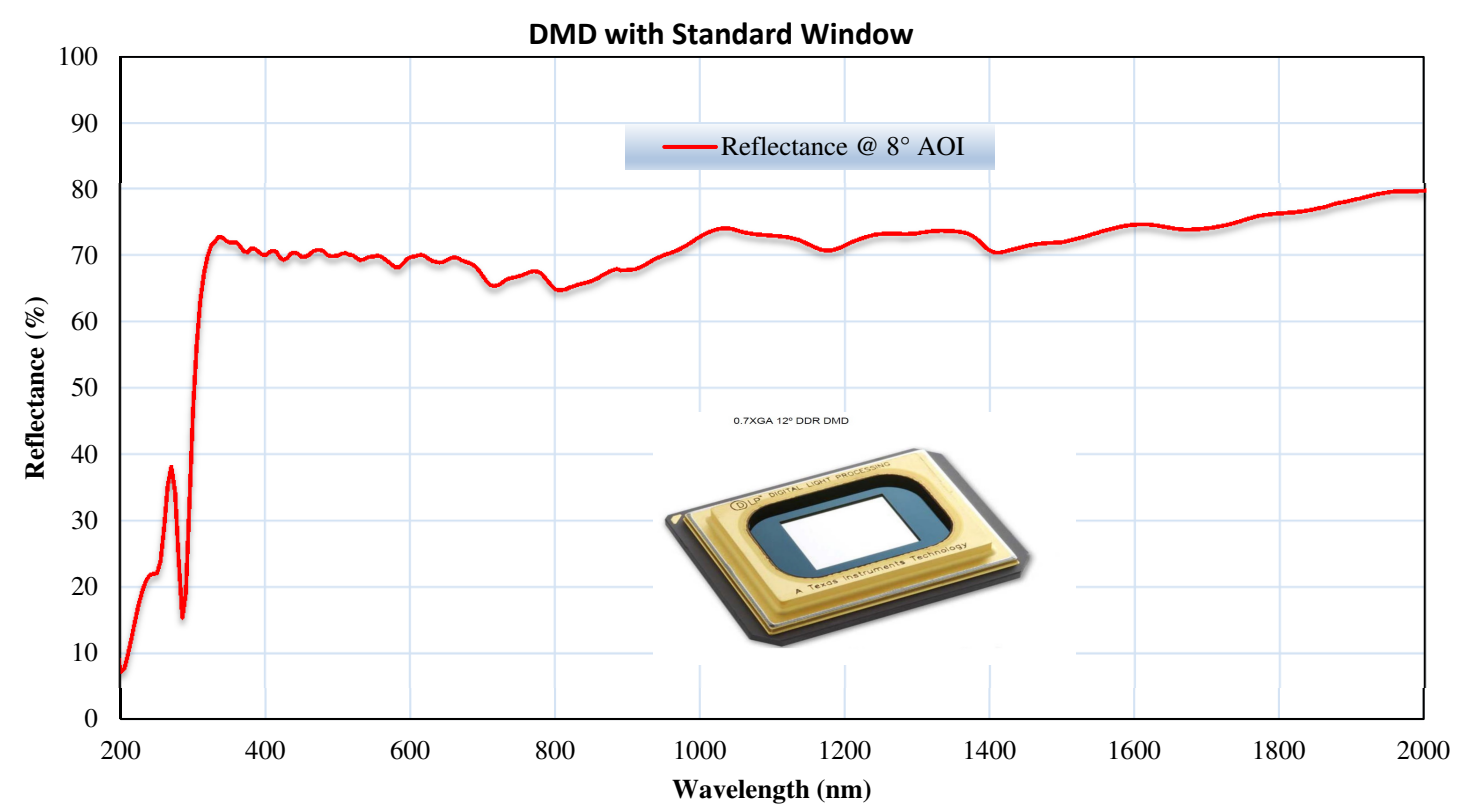

Figure 1. Near-normal reflectance of DMD chipset with standard window.

slit spectrograph today that has such a wide spectral range let alone with access to the UV. Texas Instruments (TIs) Digital Multi-Mirror Device (DMD) would make an excellent slit selector for a spectrograph. ${ }^{2,3}$ In fact, DMDs offer real promise as an efficient, inexpensive, and reliable, programmable slit mechanism for multi-object spectroscopy. One major impediment to using DMDs for UV surveys of galaxies is because these devices do not have windows that transmit light in the UV $(<380 \mathrm{~nm})$ spectral region.

The purpose of this manuscript is to report recent progress made through a NASA Strategic Astrophysics Technology (SAT) grant for performing the task of retro-fitting commercially available DMD's with UV-transmitting windows that would operate down to at least $200 \mathrm{~nm}$.

\section{DMD DESCRIPTION}

It has been shown that DMD would be ideally suited as programmable slit masks for ground-based and space telescopes to provide target selection in an astronomical instrument spectrograph. ${ }^{4}$ The task of making these devices with sensitivity in the UV $(200-400 \mathrm{~nm})$ consists first in procuring DMD chips of the 0.7 XGA Chipset type (TI part \# DLP7000). These devices have a diagonal dimension of 0.7 inch and they consist of a $1024 \times 768$ array of aluminum-based micromirrors that are digitally controlled micro-electromechanical system (MEMS) that can be used as spatial light modulator (SLM). When coupled to an appropriate optical system, these devices can be used to modulate the amplitude, direction, and phase of incoming light. The pitch or size for each micromirror is about $13.7 \mu \mathrm{m} \times 13.7 \mu \mathrm{m}$ with a tilt angle capability (relative to the flat or $0^{\circ}$ configuration) of $\pm 12^{\circ}$. The micromirror array fill factor (over the chip active area) is about $92 \%$ and the diffraction efficiency is $86 \%$. Each device is hermetically sealed and is designed for broadband visible light addressing capability. In this study, the XGA Chipsets were picked for the window replacement exercise because they are less expensive DMDs that can be operated with a TI-supplied electronics (Discovery Kit). Other than having a smaller array of mirrors, these smaller DMDs are identical to the Cinema DMDs (e.g. same mirror pitch $(13.7 \mu \mathrm{m})$ and tilt angle of $\pm 12^{\circ}$ ) with the difference that they have a larger $4 \mathrm{k}$ format which will be more suitable for use in an UV-capable spectrograph.

Figure 1 displays the normalized reflectance from the active area of a DMD device with its original window. The instrument used for collecting these data was a Perkin Elmer (model \# Lambda 950) equipped with an Universal Reflectance Accessory (URA) system. The beam foot-print produced by the spectrometer on the device occupies the size of many micromirrors (or pixels) and it has a rectangular shape of about $220 \times 360$ pixels. The 


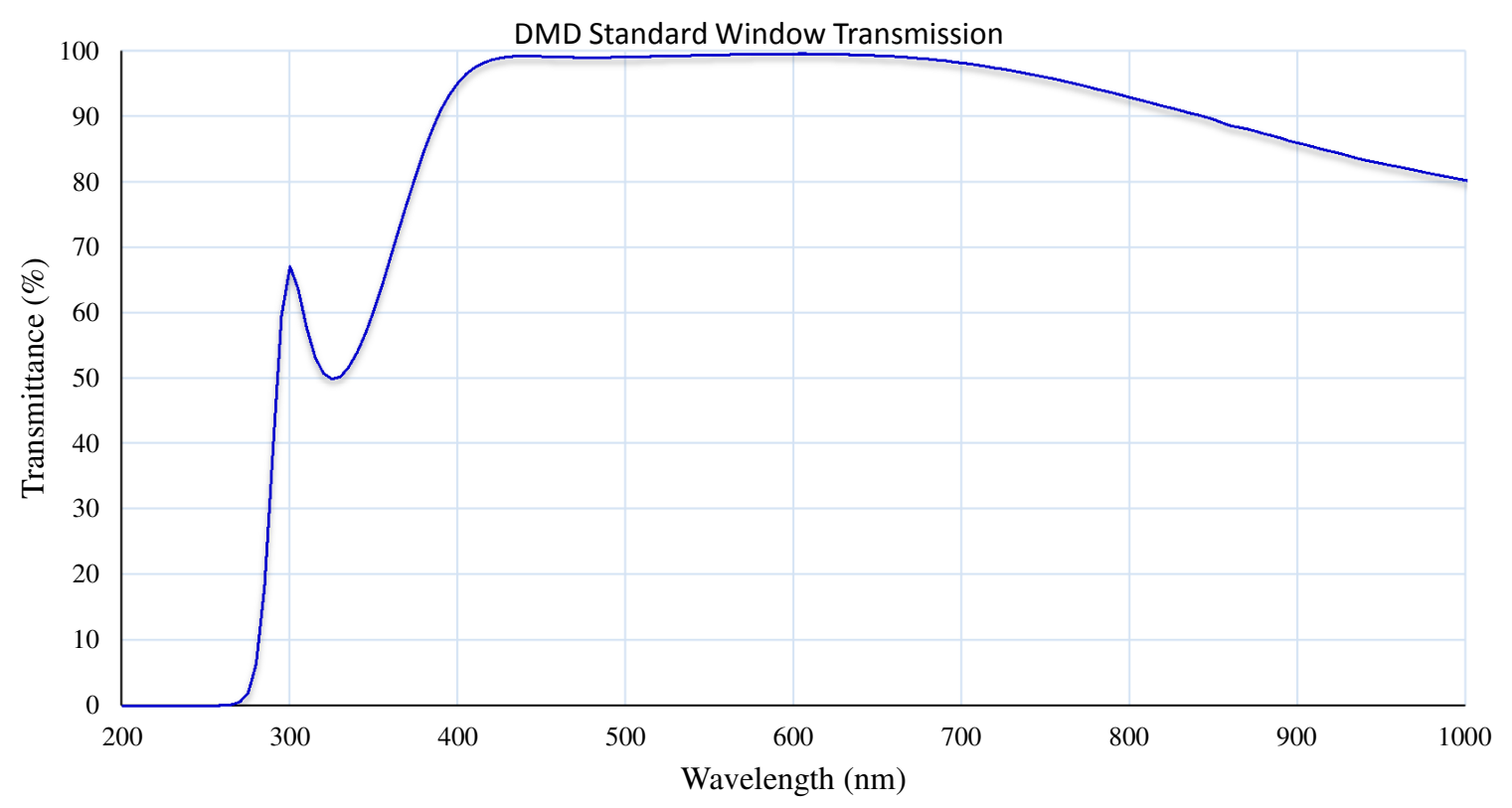

Figure 2. Normal angle of incidence transmittance for a window substrate previously removed from a DMD chipset.

device was set in passive mode (i.e. without electronic control) with all the micromirrors in their default positions (tilt angle around $0^{\circ}$ ). The angle of incidence (AOI) for these specular reflectance measurements was set at $8^{\circ}$, which was relative to the vector perpendicular to the micromirrors surface in their default flat positions. We observe the average reflectance is around $73 \%$ over the $325-2000 \mathrm{~nm}$ range. This average reflectance represents the absolute throughput of the device and it includes any losses from the intrinsic reflectance of each micromirror, the double pass through the window, and the fill factor $(\approx 92 \%)$ of the micromirrors array. These data also show a reflectance drop below $325 \mathrm{~nm}$ that is due to the window becoming opaque or absorbing for $\lambda<325 \mathrm{~nm}$. The average reflectance below $325 \mathrm{~nm}$ is around $25 \%$ and it results from reflections of the window front-surface only. This fact makes the DMD active area inaccessible below this wavelength. The only way to make the DMDs usable as a light modulation device in the UV spectral region is by replacing the existing windows with a UV-transmissive substrate. Section 3 below describes the window selection process for making UV-sensitive DMDs.

\section{UV-TRANSMISSIVE WINDOW SELECTION}

\subsection{Standard DMD Windows Characteristics}

Current DMD windows assemblies are constructed of a Kovar (ASTM-F-15) metal alloy frame fitted with a borosilicate window substrate (Corning 7056). This type of glass has been chosen because it has good transmission in the visible spectral range. ${ }^{5}$ In addition, the Corning 7056 glass has a coefficient of thermal expansion (CTE) that is very close to that of Kovar (Corning 7056 : $5.15 \times 10^{-6} /{ }^{\circ} \mathrm{C}$, vs. Kovar: $5.2 \times 10^{-6} /{ }^{\circ} \mathrm{C}$.) This good match in $\mathrm{CTE}$ allows for a glass-to-metal hermetic seal when both are heated to nearly 1000 degrees Celsius. The hermetic seal is formed by heating both the glass and metal until a wetting of the metal by the glass occurs, followed by the development of a chemical bond or some mechanical interlocking, thus maintaining the seal. The base transmission spectrum of Corning 7056 is nearly flat $(\approx 93 \%)$ throughout the visible and near-infrared spectral ranges. Figure 2 shows the transmission of a window that was previously removed from an existing DMD. The transmission shown in this figure indicates an average value close to $99 \%$ in the $400-700 \mathrm{~nm}$ range. These data indicate this window has been treated with an anti-reflection (AR) coating for optimal transmission in the visible range. This figure also shows that a DMD suited with this window would have an average transmission of $68 \%$ between $300-400 \mathrm{~nm}$, while the window is completely opaque $(T \sim 0)$ below $260 \mathrm{~nm}$. Thus, the only way to 


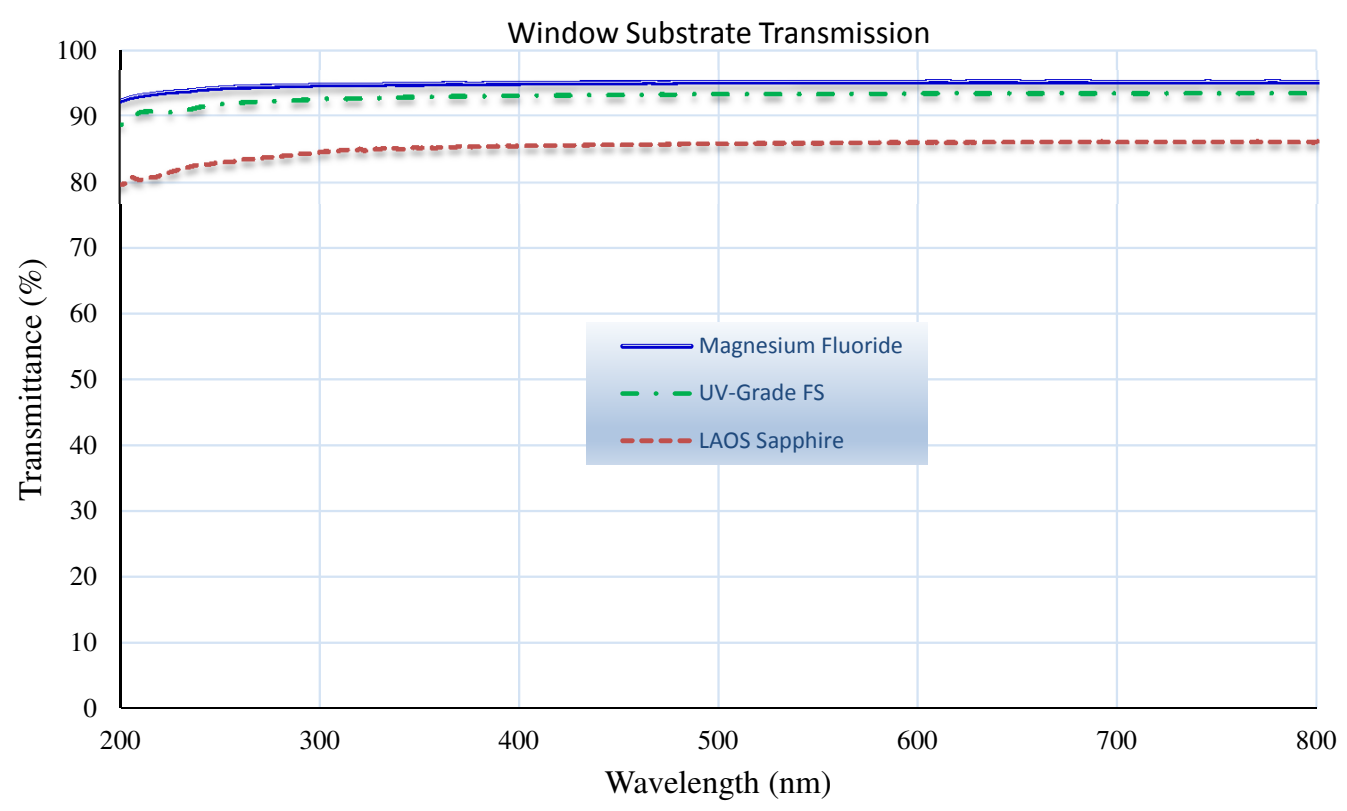

Figure 3. Transmittance at a normal angle of incidence for various UV-grade substrate options for DMD windows.

implement the DMD for application in a UV-capable spectrograph is to use a window optimized for maximum transmission in the $200-400 \mathrm{~nm}$ range.

\subsection{UV-Window Selection}

As shown in Fig. 2, standard windows used on commercially available DMD will not pass light below $250 \mathrm{~nm}$. This will preclude using these devices for application at wavelengths where the windows is required to have as low absorption as possible. Fused silica is one of the most commonly used materials from deep-UV to mid-UV wavelength applications. Due to the polycrystalline nature of this material, its physical, thermal, dielectric and optical properties are uniform and independent of crystallographic directions. The type of fused silica that would be suitable for enabling UV application of DMD devices is termed excimer or UV-grade. Unfortunately, the CTE of UV-grade fused-silica $\left(0.55 \times 10^{-6} /{ }^{\circ} \mathrm{C}\right)$ is roughly an order of magnitude smaller than that of Kovar. These dissimilar values in CTE will imply that as temperature would change during the post manufacturing process, the hermetic seal between the window and frame will not be maintained. A leaky seal will allow humidity in the environment to penetrate inside the device and this will become detrimental to the micromirrors inside causing them not to function properly. One of the most common problems is that the mirrors would become sticky and cause them not to respond to commands.

Other window alternative with good transmission in the UV is magnesium fluoride $\left(\mathrm{MgF}_{2}\right)$. This substrate is a low-index materials that transmits well into the vacuum ultraviolet (VUV) region at Lyman-Alpha $(\lambda=121.6$ $\mathrm{nm})$. For this reason, this material has wide use in applications that span from the FUV to infrared wavelengths. The crystallographic structure of $\mathrm{MgF}_{2}$ is tetragonal with a CTE that is anisotropic: $6.23 \times 10^{-6} /{ }^{\circ} \mathrm{C}$ and $10.86 \times 10^{-6} /{ }^{\circ} \mathrm{C}$ in the directions perpendicular $(\perp)$ and parallel $(\|)$ to the $c$-axis respectively. The anisotropy in CTE will require that the crystallographic axes of the $\mathrm{MgF}_{2}$ would have to be chosen carefully so that the plane of the substrate is $\perp$ to the $c$-axis given that the CTE in this perpendicular direction is a much closer match to that of the Kovar frame.

A third option in window material for use in the UV is sapphire $\left(\mathrm{Al}_{2} \mathrm{O}_{3}\right)$. The sapphire material, which is considered a medium-index substrate, is widely used as an optical window material in the UV, visible, and near infrared, mainly for its extreme toughness and strength. For this and other reasons, sapphire is also used for precision mechanical parts. Because of its hexagonal crystal structure, the CTE of crystalline sapphire is also anisotropic: $7.0 \times 10^{-6} /{ }^{\circ} \mathrm{C}$ and $7.7 \times 10^{-6} /{ }^{\circ} \mathrm{C}$ in the perpendicular and parallel directions to the $c$-axis 


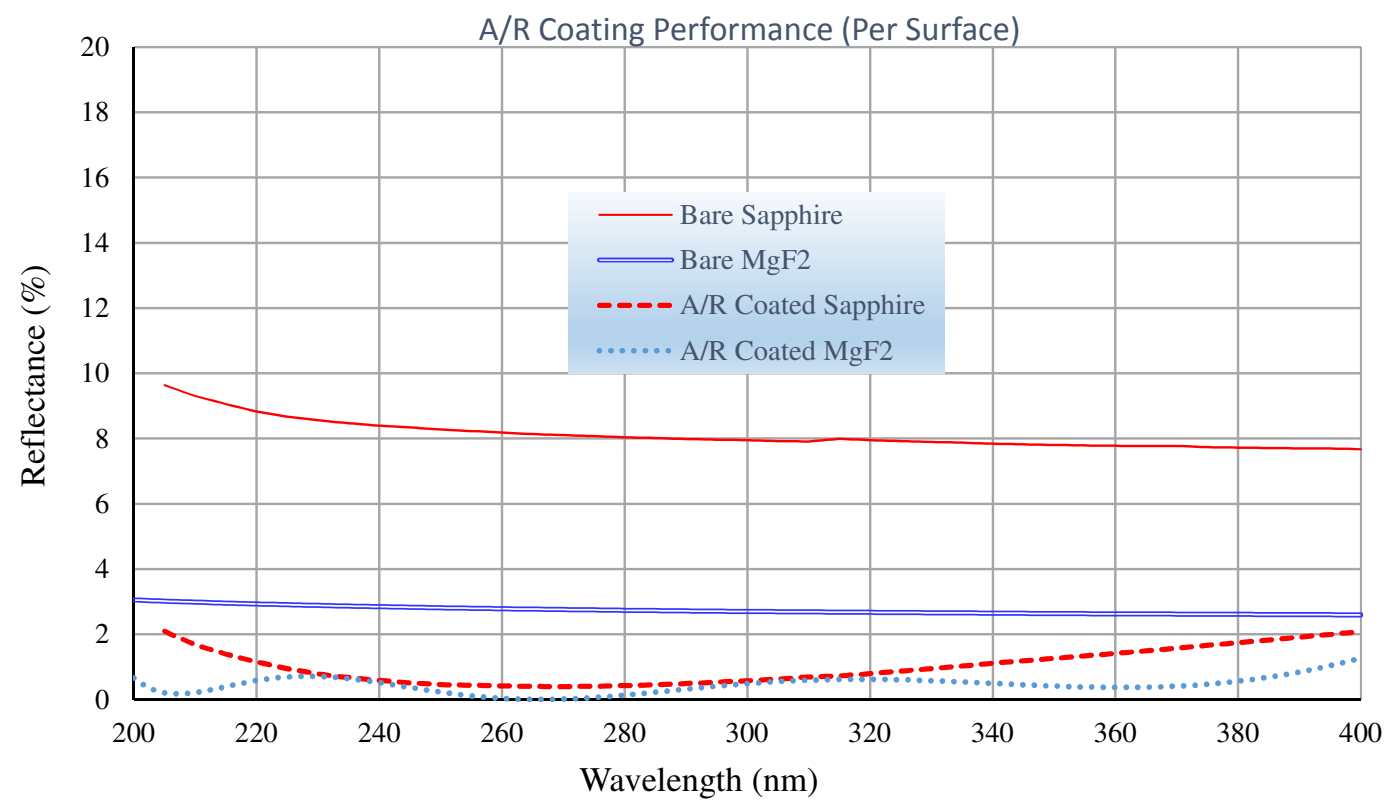

Figure 4. Antireflection coating performance for sapphire and $\mathrm{MgF}_{2}$ substrates.

respectively. Hence, the optimum choice would be to use sapphire as an optical window that would have its $c$-axis perpendicular to the plane of the substrate (because of the closer match to the Kobar CTE). A second important consideration is the optical grade of the material. Standard grade sapphire would absorb light below about $260 \mathrm{~nm}$. However, there is a low absorption optical sapphire (LAOS) material that is labeled VUV-grade. This type of sapphire combines high purity with extremely low defect density and special processing steps that results in a material that transmits light at wavelengths as low as $200 \mathrm{~nm}$, where standard sapphire material absorbs the light. The VUV grade of sapphire is especially resistant to solarization and damage from radiation and the effects of long term immersion in high power UV light.

Figure 3 shows the transmission of the three substrate materials considered above as windows alternatives to the DMD. The transmittance data shown in this figure indicate that any of the three substrate options discussed above would be acceptable alternatives to replace the window so that the device would have a window with UVtransmission capabilities. The substrate with the highest transmittance is $\mathrm{MgF}_{2}(\approx 95 \%)$ on account of having the lowest refractive index of the three. The next substrate with the highest transmittance is UV-grade fused silica (90-93\%), followed by LAOS sapphire (80-86\%). It is obvious that the transmission of these windows would have to be as high as possible in order to prevent any losses and, hence, maintain good efficiencies. Another reason is that any reflection, particularly on the inside of the window, can bounce back and forth from the inside of the window to the micromirrors to create "ghost images" that can cause interference problems with the device. The solution is to coat both sides of the window with an AR coating that is optimized for the intended wavelength range of use. Figure 4 displays the predicted performance on an AR coating applied to the surfaces of sapphire and $\mathrm{MgF}_{2}$ substrates. The AR-coating design used to suppress reflection losses on both of these substrates consists of two pairs of a high-index $\left(\mathrm{GdF}_{3}\right)$ and low-index $\left(\mathrm{MgF}_{2}\right)$ layer stacks with quarter-wave thicknesses that are optimized in the 200-400 range. Notice the reflection losses in both cases are brought down to an average of less than $1 \%$.

\section{DMD REFLECTANCE}

There is an additional consideration with respect to the use of DMDs as a programmable slit mask in the UV spectral range. It has to do with the intrinsic reflectance of the DMD active area. The commercially available DMDs are made of micromirrors based on an aluminum (Al) alloy. ${ }^{6}$ To examine the intrinsic reflectance of the micromirrors, we removed the window and measured the specular reflectance of the bare device over the 200-2000 


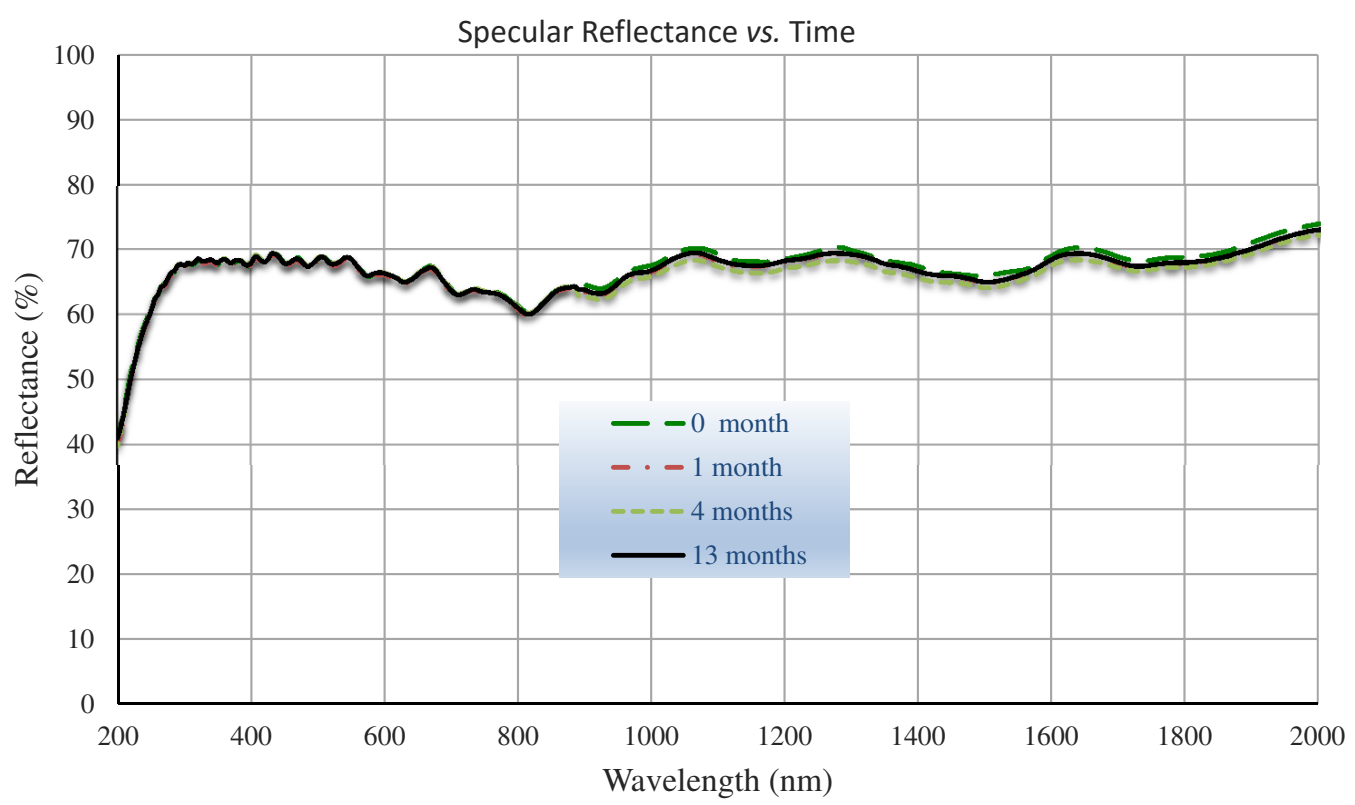

Figure 5. Specular reflectance of one DMD as a function of time. The "0 month" trace corresponds to data taken inmediately after window removal.

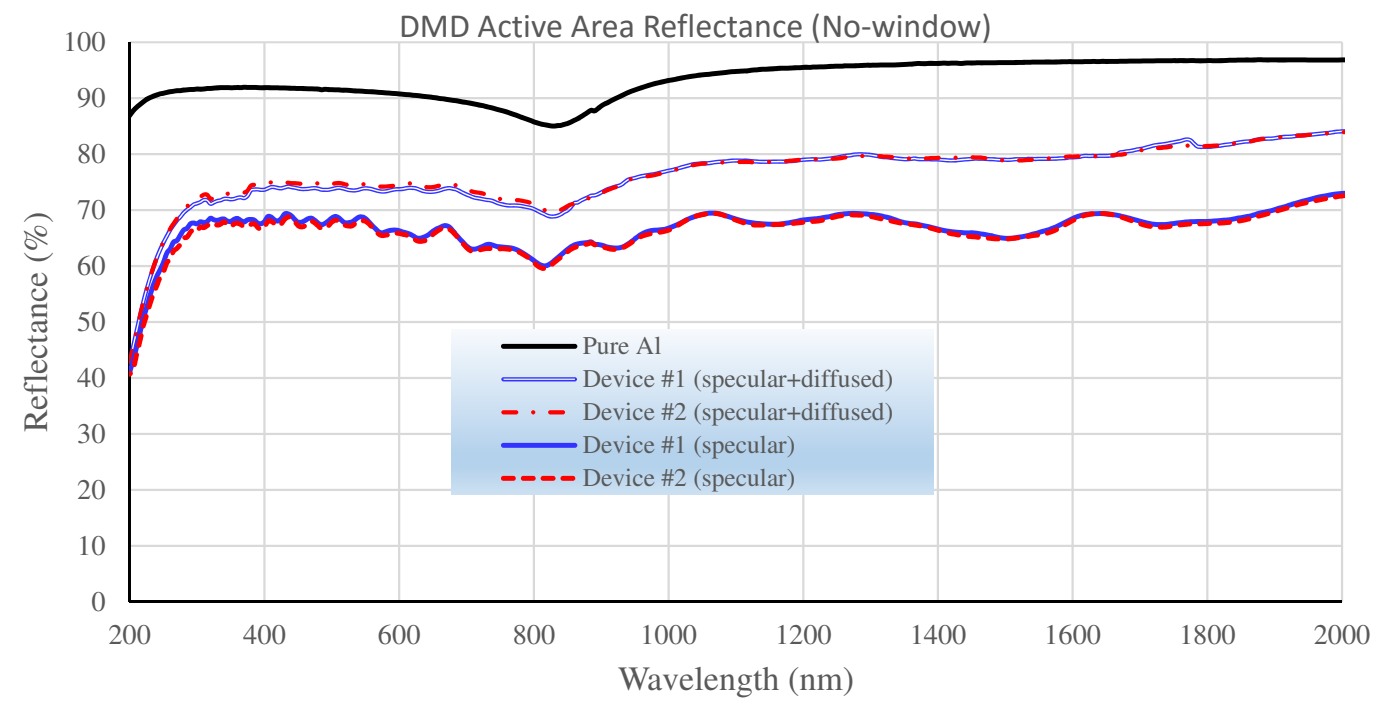

Figure 6. Specular and specular+diffused reflectance of two DMDs. The reflectance of pure Al is also shown for comparison.

nm range and using the same Perkin Elmer spectrometer fitted with the URA as described in Sec. 2. The results of these measurements are shown in Fig. 5. This figure shows the specular reflectance as a function of time (AOI $=8^{\circ}$ ) after the window has been removed and the bare device has have been exposed to ambient conditions. We first observe that the reflectance does not show any degradation over a period of more than year. However, the absolute reflectance values appear much lower than what is expected from Al-based mirrors, particularly for wavelengths lower than $300 \mathrm{~nm}$. In order to further quantify the reflectance properties of these DMDs, we show in Fig. 6 the reflectance of two bare devices (to illustrate variability) along with the reflectance of a pure Al sample. The reflectance of the two DMDs (curves labeled as "specular") is on average 35\% lower than that of the Al sample (for $\lambda>300 \mathrm{~nm}$ ) and $20 \%$ lower for longer wavelenghts. Part of these reflectance losses could be accounted for by the filling factor $(\approx 92 \%)$ due to the spaces or gaps between the micromirrors. These losses 


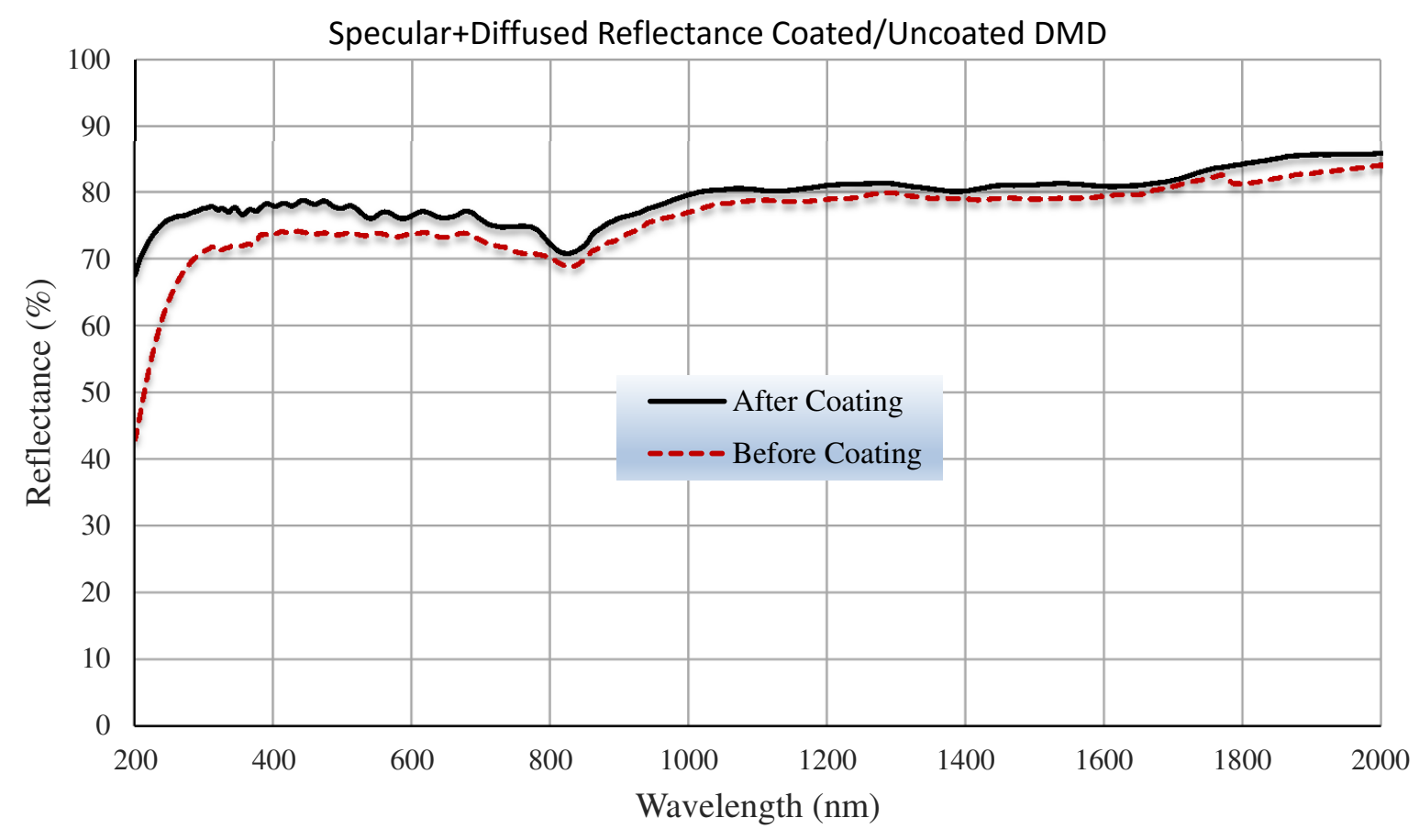

Figure 7. Specular+diffused reflectance of DMD before and after an Al coating application of $59.5 \mathrm{~nm}$.

would be realized by diffraction effects due to the periodic arrays of the micromirrors. In order to account for any losses due to these diffraction effects, we also performed Total Hemispherical Reflectance (THR), by using a $60 \mathrm{~mm}$ integrating sphere accessory attached to the Perkin Elmer spectrometer. The THR measurements include both specula plus diffused light that may be diffracted by the DMD arrays. The results of the traces labeled "specular+diffused" in Fig. 6 indicate that the THR is indeed higher than the ones corresponding to the specular component alone. But even in this case, the THR data from these devices still do not match the results obtained from the pure $\mathrm{Al}$ sample. The overall reduced reflectance of the these devices (compared to $\mathrm{Al}$ ) could be reasonably attributed to the fact that the $\mathrm{Al}$ in the DMDs may not be pure, but rather based on some type of alloy. This could be inferred by the much lower reflectance ( $42 \%$ and $41 \%$ for Devices 1 and 2 respectively) at $\lambda=200$ when compared to the reflectance of the pure $\mathrm{Al}$ sample.

\subsection{Coating of DMD Active Area}

The low reflectance of these DMDs below $300 \mathrm{~nm}$ (even when replacing the window with a substrate that has high transmittance in the UV spectral region) would severely limit the use of DMDs as programmable slit masks in a spectrograph with UV capability. One possibility to boost performance would be to coat the DMD active area with a fresh $\mathrm{Al}$ layer. In order to determine the feasibility of such approach, we carried out a coating task of applying an Al layer with thickness between 50-60 $\mathrm{nm}$ on the Device \#1 whose reflectance is shown in Fig. 6. The first step in the process was to manufacture a mask with proper dimensions that would be placed on the front of the device during the coating process. This mask would have an aperture in the middle that would match the form factor $(1024 \times 768$ pixels $)$ of the device active area. The device would then be introduced inside a high-vacuum coating chamber where the $\mathrm{Al}$ deposition would take place. The $\mathrm{Al}$ coating process is based on a Physical Vapor Deposition (PVD) method where the Al is placed in a resistive bowl (made out of Tungsten) with electrical wires attached to it. A current is passed through this bowl until the Al melts and evaporates onto the DMD inside the vacuum chamber. The device with the mask were placed directly on top of the bowl for the $\mathrm{Al}$ evaporation. One parameter that determines the quality of the deposited $\mathrm{Al}$ is a high vacuum (which was maintained from the low $10^{-7}$ Torr to the high $10^{-8}$ Torr range) during the coating process. We also ensured this vacuum chamber would have very low levels of residual water vapor and oxygen in order to minimize the possibility of oxidation of the $\mathrm{Al}$ layer. The $\mathrm{Al}$ deposition rate was maintained at $100 \AA$ per 


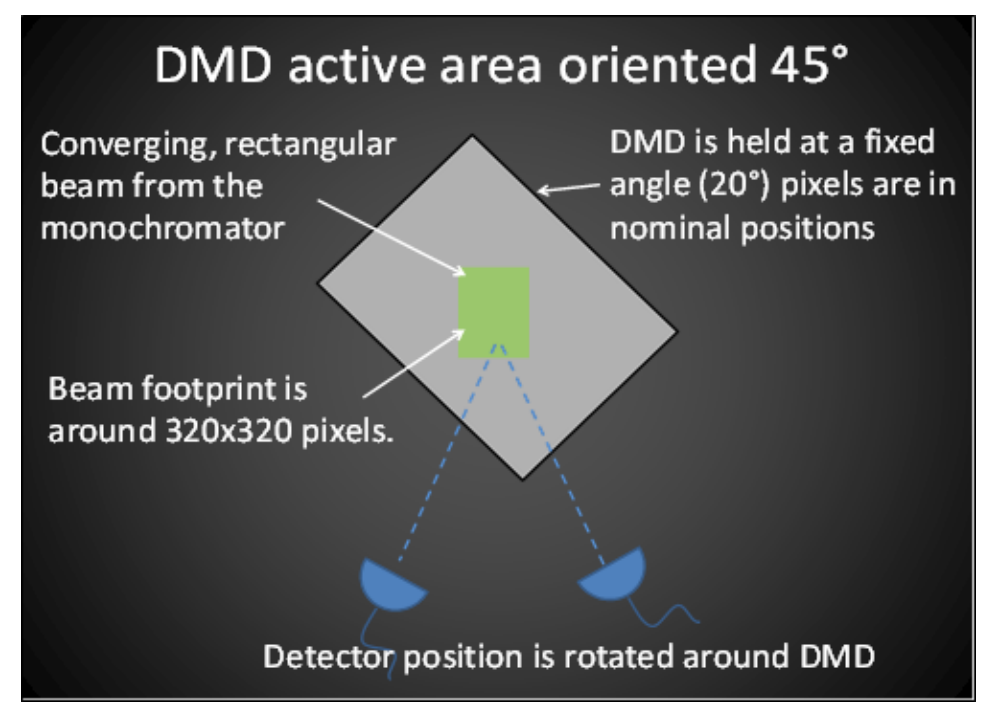

Figure 8. Setup for measuring reflectance of bare DMD.

second or higher, in order to obtain a denser $\mathrm{Al}$ film. Figure 7 displays the THR reflectance (specular+diffused) before and after $\mathrm{Al}$ was applied on the DMD active area. The coated device shows a substantial increase in reflectance reaching a value of $68 \%$ at $200 \mathrm{~nm}$, when compared to the value prior to the coating application $(42 \%)$. The results also show a more modest (4-5\%) increase across the 400-2000 $\mathrm{nm}$ wavelength range, and this may suggest the type of $\mathrm{Al}$ from which the micromirrors are made may contain some type of alloy that exhibits a lower reflectance than that of pure Al. A topic of a future investigation will be to determine the effect of the evaporated $\mathrm{Al}$ on the functionality of the DMD. The main reason is because the deposited $\mathrm{Al}$ would go not only on the micromirrors surface, but also has a small but distinct possibility of being deposited in-between the micromirror gaps and the surfaces behind them. The biggest risk from the Al deposition would be an electrical short to the electromechanical mechanism that tilts each micromirror into the $\pm 12^{\circ}$ states.

\subsection{DMD Scattering Properties}

We will now discuss the scattering performance of the DMD micromirror arrays. These measurements were made possible by using a Cary5000 spectrometer that is equipped with an Universal Measurement Accessory (UMA). The UMA defines two independent angular coordinate systems to describe the positions of the sample and detector with respect to the incident light beam on the sample. The sample angle, which is defined as the angle between the direction of the incident beam and the direction perpendicular to the device surface, can be fully driven from $0^{\circ}$ through $360^{\circ}$. On the other hand, the detector angle can be set in the range of $10^{\circ}-180^{\circ}$ on either side of the incident beam. The specular reflectance of the sample is obtained when, for a given sample angle $\theta_{s}$, the detector angle is set at $2 \theta_{s}$. Figure 8 shows how the DMD was configured inside the UMA sample compartment. This figure shows the DMD is rotated in-plane by $45^{\circ}$ so that the diagonals of each micromirror are either in the horizontal or vertical directions. This figure also shows a sketch of the beam footprint the UMA produces on the device active area that covers an area of approximately $320 \times 320$ pixels or micromirrors. The DMD was not powered up for these scattering measurements so the exact position of individual DMD mirrors is not exactly determined and this uncertainty contributes to additional scattering. Figure 9 displays how the DMD reflectivity changes as the detector angle is varied from $10^{\circ}$ through $90^{\circ}$ along two directions within the plane of the DMD with the device AOI fixed at $20^{\circ}$. This figure shows that, as expected, the DMD produces a mostly specular reflectance where the peak reflectance is reached at a detector angle of $40^{\circ}$. Secondly, this figure shows a marked difference in the reflectance baseline (away from the specular component) when the detector is scanned along two different orientations on the DMD (the curves labeled as " 0 " " and " $45^{\circ}$ "). It should be noted that the inset of Fig. 9 shows a strong diffraction pattern along the micromirror gap directions, which are along the $\pm 45^{\circ}$ directions (relative to the horizontal or $0^{\circ}$ orientation) in the image. As mentioned earlier, these diffraction 


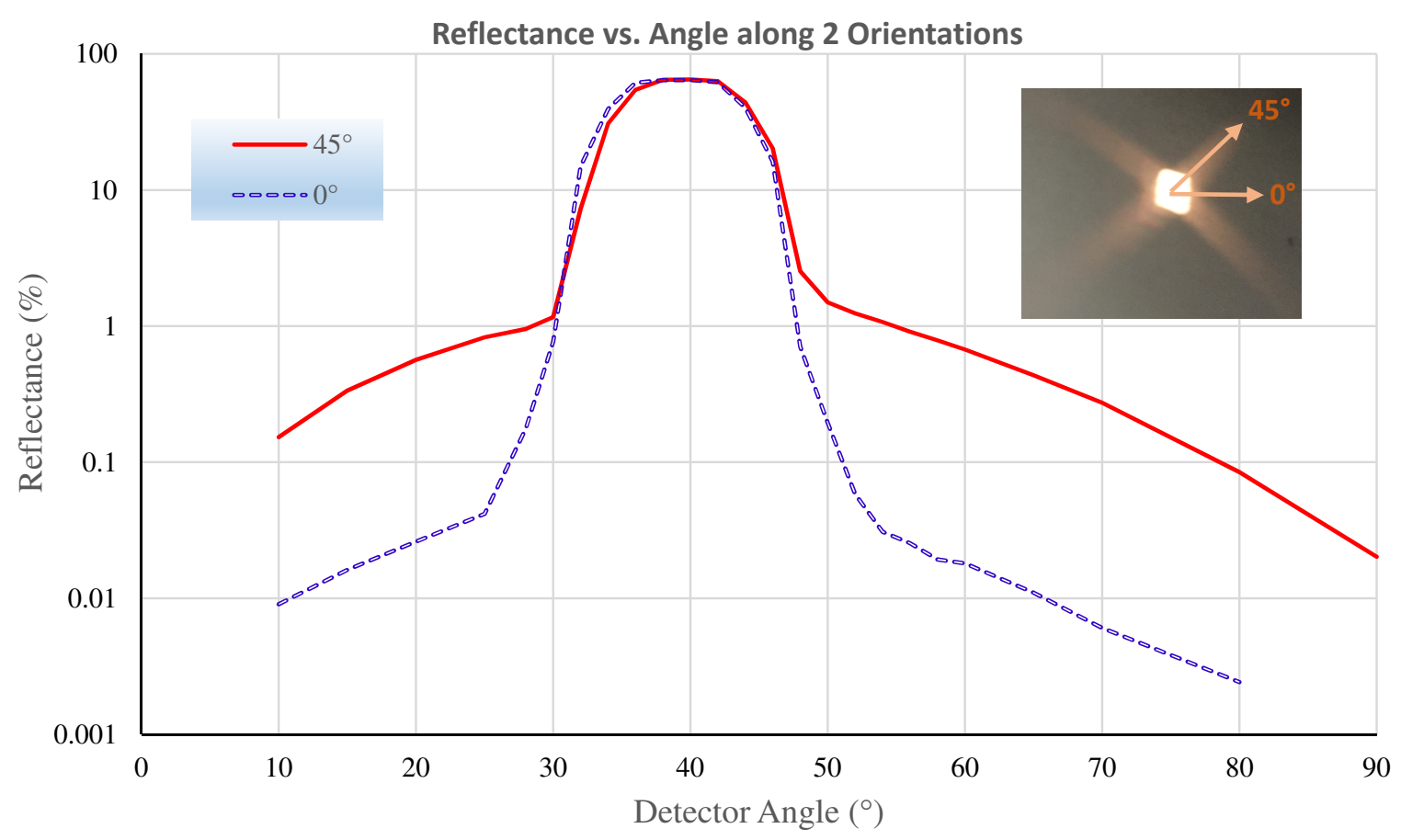

Figure 9. Reflectance vs. angles for two orientations on the DMD. The inset in this figure illustrates the diffraction pattern along the $\left(\theta= \pm 45^{\circ}\right)$ directions when micromirrors diagonal is along horizontal (or vertical) directions. Notice these diffraction patterns are formed when light illuminates the square arrays of micromirrors which are separated by a gap of $0.6 \mu \mathrm{m}$.

DMD XGA Chip with Original Window $\mathrm{AOI}=20^{\circ}$

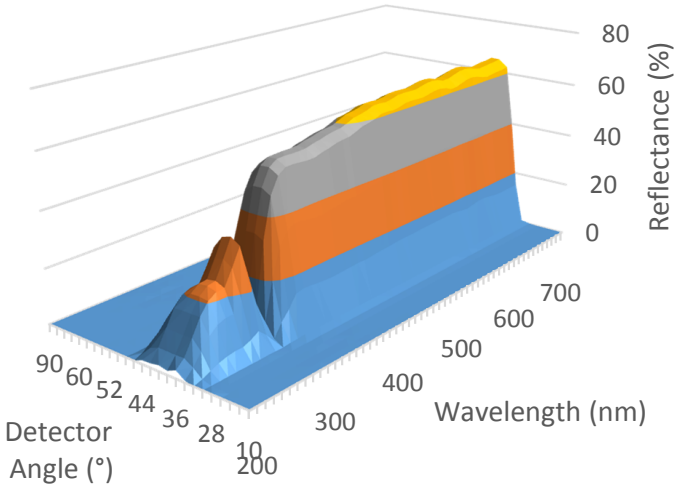

DMD XGA Chip without window (Al-Coated) $\mathrm{AOI}=20^{\circ}$

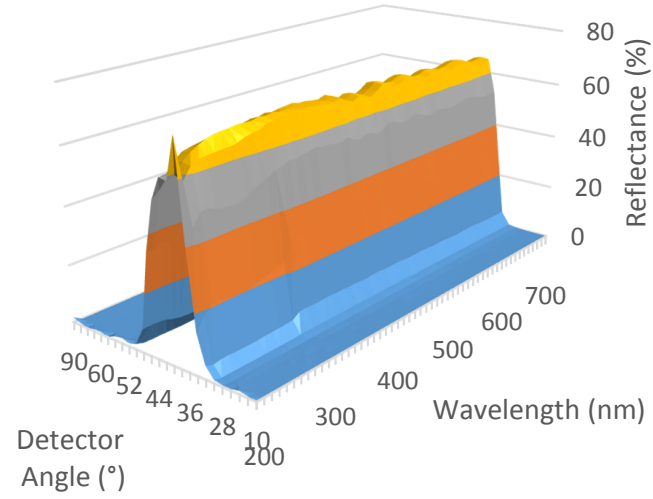

Figure 10. Sample and detector angle definitions in the Cary5000+UMA system.

patterns are caused by the micromirror gap separation being in the order of visible or IR wavelengths $(0.6 \mu \mathrm{m})$. Hence, the data shown in Fig. 9 indicates an 1-2 order of magnitude increase in the reflectance baseline when detector is scanned along the diffraction pattern directions when compared to scanning along the horizontal (or vertical) directions that do not contain the diffraction pattern. Figure 10 displays two 3-dimensional plots of the reflectance as a function of detector angle and wavelength for the same device. The graph on the left shows the reflectance performance for the device with the standard (borosilicate) window, while the plot on the right shows the reflectance performance of the same device after the window was removed and the DMD was coated with an $\mathrm{Al}$ layer as discussed in Sec. 4.1. These figures show more clearly the gains in reflectance that are realized after 
the device has been coated with the Al layer.

\section{CONCLUSIONS}

In conclusion, we evaluated UV-transmitting windows made out of UV-grade fused silica, $\mathrm{MgF}_{2}$, and LAOS sapphire that could be used to replace standard borosilicate windows on commercially available DMDs in order to enable these devices as programmable slit masks with UV capabilities. Secondly, we performed specular and diffused reflectance on DMDs which had their windows removed in order to determine the throughput, durability, and scattering properties of the micromirrors reflective area. We found that although the reflectivity of bare devices was very stable and did not change over a period of more than year, the base reflectivity was low $(\approx 58 \%$ over the 200-300 nm range). Because this reflectivity was lower than that of pure $\mathrm{Al}$, we performed a coating experiment where the DMD active area of one device was coated with a fresh layer of pure $\mathrm{Al}$ (thickness $=59.5$ $\mathrm{nm}$ ). We observed the average reflectance of the newly coated device increased to an average value of $75 \%$ in the 200-300 spectral region. This boost in reflectance along with the replacement of the window with one of the three substrate options mentioned above, will provide a viable path to use the DMD as a programmable slit mask in the UV spectral range. Future tasks will include verifying that Al-coated DMD will remain fully functional and that no electrical short would occur due to the $\mathrm{Al}$ deposition on the electronic mechanism that provides the $\pm 12^{\circ}$ micromirror tilt. Additional studies will also include a verification that adding this Al layer would not introduce changes in the DMD wavefront surface figure error distortion that may be induced by changes in the flatness of the micromirrors array.

\section{ACKNOWLEDGMENTS}

This work has been performed in the Optics Branch (Code 551) at the Goddard Space Flight Center (GSFC) and supported through a NASA SAT grant No. NNX14AI62G. The authors wish to thank Linette Kolos and Felix Threat (NASA-GSFC/551) for their support in performing Al deposition on the bare DMD.

\section{REFERENCES}

[1] S. Jouvel, et.al.., "Designing future dark energy space missions. building realistic galaxy spectro-photometric catalogs and their first applications," Astronomy \& Astrophysics 504, 359 (2009).

[2] Kearney, K. and Ninkov, Z., "Characterization of a digital micromirror device for use as an optical mask in imaging and spectroscopy," in [Spatial Light Modulators], Sutherland, R., ed., Proceedings of the Society of Photo-Optical Instrumentation Engineers (SPIE) 3292, 81-92 (1998).

[3] Fourspring, K., "Assessing the performance of digital micromirror devicestm for use in space-based multiobject spectrometers," (2013).

[4] F. Zamkotsian, P. Lanzoni, E. Grassi, R. Barette, C. Fabron, K. Tangen, L. Valenziano, L. Marchand, L. Duvet, "Successful evaluation for space applications of the 2048x1080 dmd," in [Emerging Digital Micromirror Device Based Systems and Applications III], Douglass, M. R., ed., Proceedings of the Society of Photo-Optical Instrumentation Engineers (SPIE) 7932, 79320A (2011).

[5] [Wavelenght Transmittance Considerations for DLP DMD Window], Texas Instruments, http://www.ti.com/lit/an/dlpa031c/dlpa031c.pdf (May 2012-Revised March 2014).

[6] B.M. Callies, et.al., "Digital micromirror device having a window transparent to ultraviolet (uv) light," $U$. S. Patent no: 7,161,727 B2 (2007). 